\title{
Multiplicity of Nearby Free-floating Late $M$ and L Dwarfs: HST-WFPC2 Observations of Candidates and Bona Fide Binary Brown Dwarfs
}

\author{
Hervé Bouy \& Wolfgang Brandner \\ European Southern Observatory, Karl Schwarzschildstraße 2, Garching, \\ Germany
}

\author{
Eduardo L. Martín \\ Institute for Astronomy, 2680 Woodlawn Drive, Honolulu, HI 96822, \\ U.S.A
}

Xavier Delfosse

Laboratoire d'Astrophysique de l'Observatoire de Grenoble, 414 rue de la Piscine, F-38400 Saint Martin d'Hère, France

France Allard

Centre de Recherche Astronomique de Lyon (UML 5574), Ecole

Normale Superieure, 69364 Lyon Cedex 07, France

Gibor Basri

University of California at Berkeley, Astronomy Department, MC 3411 Berkeley, $C A$ 94720, USA

\begin{abstract}
We review the results of HST/WFPC2 observations of a sample of 50 very-low-mass objects in the solar neighborhood (30 from our own program and 20 from the archive). Using high spatial resolution HST/WFPC2 images, we observed these objects in two filters (F814W and $\mathrm{F} 675 \mathrm{~W}$ ) in order to identify close companions, measure their colours, and obtain first epoch data of the newly discovered binaries. In addition to 3 previously known binaries we discovered 3 new multiple systems. Second epoch data are required to confirm that they are real common proper motion pairs. Binary properties like multiplicity, distribution of binary separations and brightness ratios hold clues on the origin and evolution of free-floating brown dwarf binaries.
\end{abstract}

\section{Sample selection and Data Analysis}

Our sample consists of 30 objects detected by the near-infrared surveys DENIS and 2MASS. In addition to this set of data and in order to increase the sample of objects we also used data from the archive (HST program GO8146). The sample of this program has $20 \mathrm{~L}$ dwarfs identified in the 2MASS survey. They were 
observed using the Planetary Camera CCD of WFPC2 in two filters (F814W and $\mathrm{F} 606 \mathrm{~W}$ ). In total we thus had 50 objects in the sample.

Nearby free-floating brown dwarfs do not form a coeval sample, but they are close enough ( $5 \mathrm{pc}$ to $30 \mathrm{pc}$ ) to measure their distance (angular parallax) precisely, and - by using HST/WFPC2 - it is possible to detect and resolve binary brown dwarfs with separations down to $0.3 \mathrm{~A} . \mathrm{U}$. $\left(0.06^{\prime \prime}\right.$ at $\left.5 \mathrm{pc}\right)$.

The multiple systems, identified either by visual inspection or measurement of ellipticity, were analyzed by PSF fitting, using PSF stars in the field. To minimize the effects due to the position sensitivity of the PSF shape in the detector and the slight change of HST focus from one orbit to another, we used 8 different PSF stars in each case, and calculated the average value and standard deviation for the uncertainties.

\section{Binary Frequency and distribution of separations}

We found 10 binaries among a sample of 50 objects. This gives an observed binary frequency of $\sim 20 \%$. This value is only indicative and not a precise physical value for several reasons. The sample is indeed not statistically well defined. The selection introduced a bias since we did not choose the targets completely randomly among field brown dwarfs: some were selected from their colours and brightnesses. Our sample is thus limited in magnitude rather than in distance. Second epoch data are required in order to confirm the multiplicity of the new candidates.

The main result of this preliminary study is that we did not find any binary with separations wider than $15 \mathrm{AU}$. This was already mentioned as the possible Brown Dwarf Wide Binary Desert. This can be explained either by differences in the formation mechanisms or by a normal evolution process of the multiple systems such as disruption due to the gravitational interaction with neighbouring stars and/or molecular clouds.

We also observed a lack of systems with large luminosity differences (see Figure 1), which might correspond to a lack of systems with unequal masses. The transformation of luminosity ratios to mass ratios is not straightforward since the ages of the various individual systems can be very different, but if we assume that our sample covers a randomly large range of ages (typically between 0.5 and $10 \mathrm{Gyrs}$ ), we can suggest that this might be evidence of a preference for equal-mass system as it was thought before.

This paper is based on observations with the NASA/ESA Hubble Space Telescope obtained at the Space Telescope Science Institute, which is operated by the Association of Universities for Research in Astronomy, Incorporated, under NASA contract NAS5-26555. Support for Proposal number 9157 was provided by NASA through a grant from the Space Telescope Science Institute. 


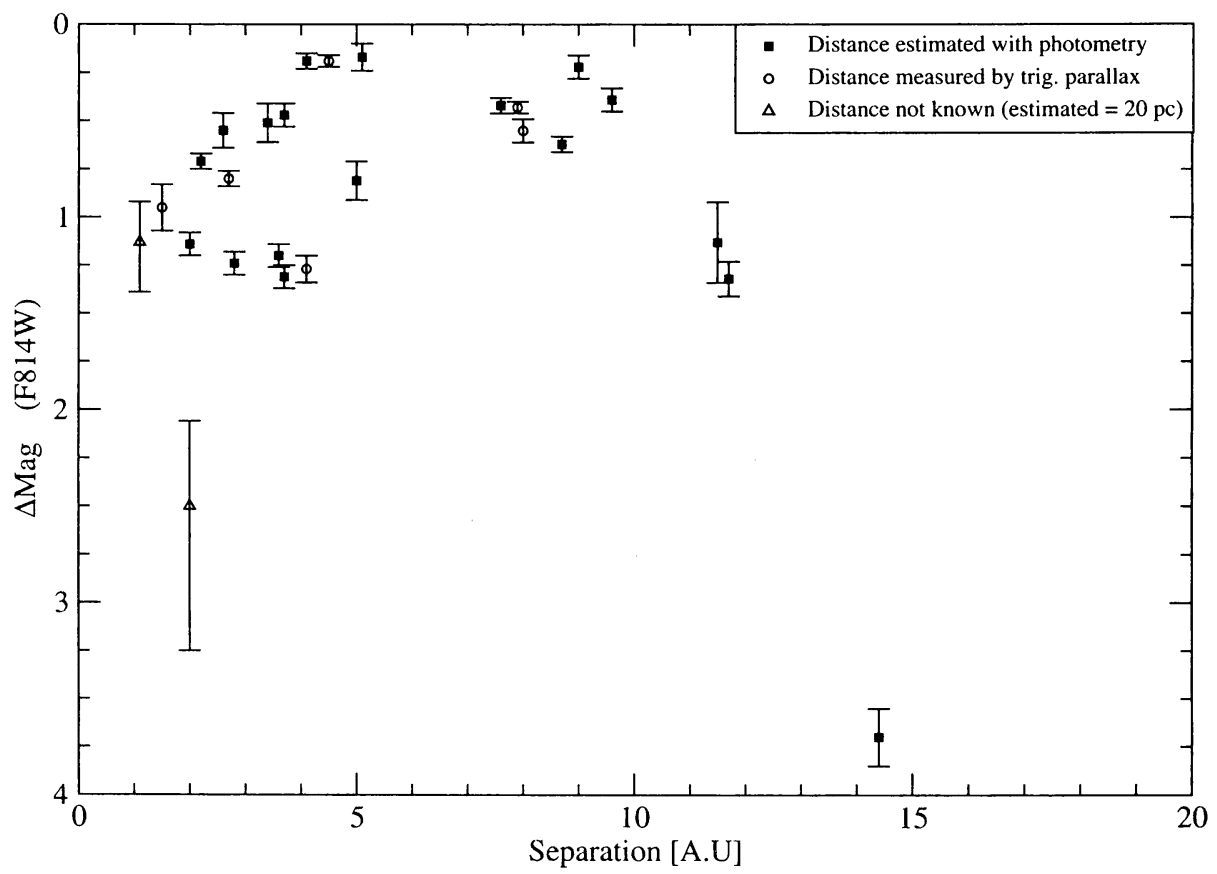

Figure 1. Difference of magnitude between the primary and the secondary as a function of the separation. There is a gap between $\Delta \mathrm{Mag}$ $\simeq 1.5 \mathrm{mag}$ and $\Delta \mathrm{Mag} \simeq 2.5 \mathrm{mag}$, suggesting that there is a preference for equal-mass systems and a lack of systems with small mass ratios. 


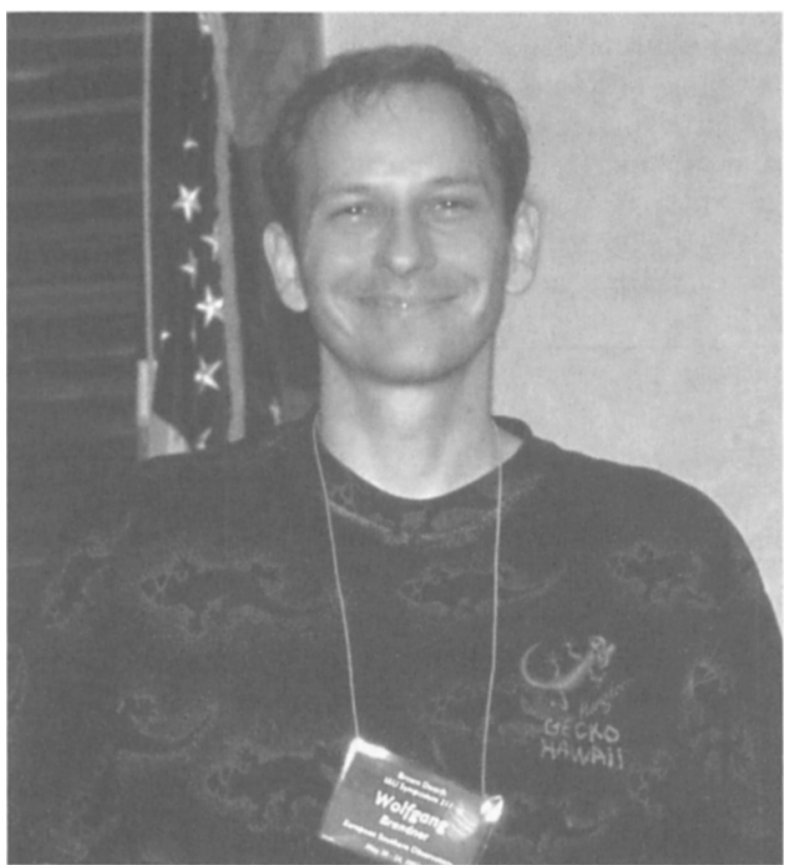

Wolfgang Brandner 